UDC 517.547

LBC 22.161 .5

\title{
A QUESTION OF AHLFORS
}

\section{Krushkal Samuel L.}

Doctor of Physical and Mathematical Sciences, Professor, Department of Mathematics Bar-Ilan University and University of Virginia slk6z@cms.mail.virginia.edu 5290002 Ramat-Gan, Israel and Charlottesville, VA 22904-4137, USA

Abstract. In 1963, Ahlfors posed in [1] (and repeated in his book [2]) the following question which gave rise to various investigations of quasiconformal extendibility of univalent functions.

Question. Let $f$ be a conformal map of the disk (or half-plane) onto a domain with quasiconformal boundary (quasicircle). How can this map be characterized?

He conjectured that the characterization should be in analytic properties of the logarithmic derivative $\log f^{\prime}=f^{\prime \prime} / f^{\prime}$, and indeed, many results on quasiconformal extensions of holomorphic maps have been established using $f^{\prime \prime} / f^{\prime}$ and other invariants (see, e.g., the survey [9] and the references there).

This question relates to another still not solved problem in geometric complex analysis:

To what extent does the Riemann mapping function $f$ of a Jordan domain $D \subset \widehat{\mathbb{C}}$ determine the geometric and conformal invariants (characteristics) of complementary domain $D^{*}=\widehat{\mathbb{C}} \backslash \bar{D}$ ?

The purpose of this paper is to provide a qualitative answer to these questions, which discovers how the inner features of biholomorphy determine the admissible bounds for quasiconformal dilatations and determine the Kobayashi distance for the corresponding points in the universal Teichmüller space.

Key words: the Grunsky inequalities, Beltrami coefficient, universal Teichmüller space, Teichmüller metric, Kobayashi metric, Schwarzian derivative, Fredholm eigenvalues.

In the memory of outstanding mathematician and excellent person Vladimir Miklyukov

\section{Background}

The underlying features are created by the Grunsky inequalities (cf. [10]). Recall that the Grunsky coefficients $\alpha_{m n}(f)$ of a univalent function $f$ in the unit disk $\Delta=\{|z|<1\}$ with $f(0)=0, f^{\prime}(0)=1$ are determined from the expansion

$$
\log \frac{f(z)-f(\zeta)}{z-\zeta}=\sum_{m, n=1}^{\infty} \alpha_{m n} z^{m} \zeta^{n} \quad\left(z, \zeta \in \Delta^{2}\right)
$$


(where the principal branch of the logarithmic function is chosen) and satisfy the inequality

$$
\left|\sum_{m, n=1}^{\infty} \sqrt{m n} \alpha_{m n}(f) x_{m} x_{n}\right| \leq 1
$$

for any sequence $\mathbf{x}=\left(x_{n}\right)$ from the unit sphere $S\left(l^{2}\right)$ of the Hilbert space $l^{2}$ with norm $\|\mathbf{x}\|=\left(\sum_{1}^{\infty}\left|x_{n}\right|^{2}\right)^{1 / 2}$ (cf. [5]).

We shall consider with the functions with quasiconformal extensions across the unit circle $\partial \Delta$. Their Beltrami coefficients run over the unit ball

$$
\operatorname{Belt}\left(\Delta^{*}\right)_{1}=\left\{\mu \in L_{\infty}(C): \mu(z) \mid D=0, \quad\|\mu\|_{\infty} \cdot<1\right\}
$$

Denote by $w^{\mu}$ the solution of the Beltrami equation $\bar{\partial} w=\mu \partial w$ on $\mathbb{C}$ normalized by

$$
w^{\mu}(z)=z+\sum_{2}^{\infty} a_{n} z^{n}, \quad z \in \Delta ; \quad w^{\mu}(\infty)=\infty .
$$

Such functions form the class $S(\infty)$; it is foliated by the equivalence classes $[f]=\left\{w^{\mu}\right.$ : : $\left.w^{\mu} \mid \Delta=f\right\}$. We shall also write $\mu \in[f]$.

Note that Beltrami equation with $\mu \in \operatorname{Belt}\left(\Delta^{*}\right)_{1}$ determines its solutions with $w(0)=$ $=w^{\prime}(0)-1=0$ up to the fractional linear transformation

$$
\sigma_{a}: w \mapsto w /(1-a w)
$$

depending on $a=1 / w(\infty)$, and $\sigma_{a} \circ w \in S(\infty)$.

The minimum $k(f)$ of dilatations $k\left(w^{\mu}\right)=\|\mu\|_{\infty}$ in the equivalence class of $f$ is called the Teichmüller norm of this function. It dominates the Grunsky norm

$$
\varkappa(f)=\sup \left\{\left|\sum_{m, n=1}^{\infty} \sqrt{m n} \alpha_{m n} x_{m} x_{n}\right|: \mathbf{x}=\left(x_{n}\right) \in S\left(l^{2}\right)\right\}
$$

by $\varkappa(f) \leq k(f)$ [12] (or even a stronger form found recently in [9], but this will not be used here).

These norms coincide only when any extremal Beltrami coefficient $\mu_{0}$ for $f$ satisfies

$$
\left.\left\|\mu_{0}\right\|_{\infty}=\sup \left\{\left|\iint_{D^{*}} \mu(z) \psi(z) d x d y\right|\right\}: \psi \in A_{1}^{2}\left(\Delta^{*}\right),\|\psi\|_{A_{1}}=1\right\} \quad(z=x+i y)
$$

where $A_{1}\left(\Delta^{*}\right)$ denotes the subspace in $L_{1}\left(\Delta^{*}\right)$ formed by holomorphic functions (hence, $\psi(z)=c_{4} z^{-4}+c_{5} z^{-5}+\cdots=O\left(z^{-4}\right)$ as $\left.z \rightarrow \infty\right)$ and $A_{1}^{2}\left(\Delta^{*}\right)$ is its subset consisting of $\psi$ with zeros even order in $\Delta$, i.e., of the squares of holomorphic functions. Moreover, if $\varkappa(f)=k(f)$ and the equivalence class of $f$ is a Strebel point (i.e., contains extremal extension of Teichmüller type $f^{k\left|\psi_{0}\right| / \psi_{0}}$ ), then $\psi_{0}=\omega^{2} \in A_{1}^{2}$ (cf. [6;9]). In the case, when the curve $f\left(S^{1}\right)$ is analytic, the evenness of zeros of $\psi_{0}$ was established by a different method in [14].

One can apply to $f \in S(\infty)$ the rotational conjugation

$$
\mathcal{R}_{p}: f(z) \mapsto f_{p}(z):=f\left(z^{p}\right)^{1 / p}=z+\frac{a_{2}}{p} z^{p-1}+\ldots
$$


with integer $p \geq 2$ which transforms $f \in S(\infty)$ into $p$-symmetric univalent functions accordingly to the commutative diagram

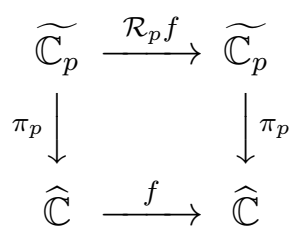

where $\widetilde{\mathbb{C}_{p}}$ denotes the $p$-sheeted sphere $\widehat{\mathbb{C}}$ branched over 0 and $\infty$, and the projection $\pi_{p}(z)=$ $=z^{p}$. This transform acts on $\mu \in \operatorname{Belt}\left(\Delta^{*}\right)_{1}$ and $\psi \in L_{1}\left(\Delta^{*}\right)$ by

$$
\mathcal{R}_{p}^{*} \mu=\mu\left(z^{p}\right) \bar{z}^{p-1} / z^{p-1}, \quad \mathcal{R}_{p}^{*} \psi=\psi\left(z^{-p}\right) p^{2} z^{2 p-2},
$$

and $k\left(\mathcal{R}_{p} f\right)=k(f), \varkappa\left(\mathcal{R}_{p} f\right) \geq \varkappa(f)$.

\section{Theorem}

Fix $0<r<1$ and consider for $\mu \in \operatorname{Belt}\left(\Delta^{*}\right)_{1}$ the maps

$$
f_{r}^{\widetilde{\mu}}(z)=r^{-1} f^{\mu}(r z), \quad z \in \mathbb{C}
$$

with Beltrami coefficients $\widetilde{\mu}(z)=\mu(r z)$. Take the truncated Beltrami coefficients

$$
\mu_{r}(z)= \begin{cases}\mu(r z), & |z|>1 \\ 0, & |z|<1\end{cases}
$$

getting a linear (hence holomorphic) map

$$
\iota_{r}: \mu \mapsto \mu_{r}: \operatorname{Belt}\left(\Delta^{*}\right)_{1} \rightarrow \operatorname{Belt}(\Delta)_{1} .
$$

Theorem 1. For any univalent function $f(z)=z+c_{2} z^{2}+\ldots$ in the unit disk $\Delta$, the following assertions are equivalent:

(a) $f$ has quasiconformal extension across the unit circle $S^{1}=\partial \Delta$ (hence, $f\left(S^{1}\right)$ is a quasicircle).

(b) $\varkappa(f)<1$.

(c) $f$ admits $k$-quasiconformal extensions across the unit circle $S^{1}$ to $\widehat{\mathbb{C}}$ with

$$
k \geq \widehat{\varkappa}\left(\sigma_{a} \circ f\right):=\sup _{\mu \in\left[\sigma_{a} \circ f\right]} \limsup _{r \rightarrow 1} \sup _{p} \sup _{\psi \in A_{1}^{2}\left(\Delta^{*}\right),\|\psi\|_{A_{1}}=1}\left|\iint_{D^{*}} \mathcal{R}_{p}^{*} \mu_{r}(z) \psi(z) d x d y\right| .
$$

This lower admissible bound $\hat{\varkappa}\left(\sigma_{a} \circ f\right)$ for quasiconformal dilatations of extensions (and thereby for the Grunsky norm of $f$ ) is sharp in the sense that it cannot be replaced by a smaller for each $f \in S(\infty)$.

(d) The Kobayashi distance between the Schwarzian $S_{f}$ representing $\left[\sigma_{a} \circ f\right]$ in the universal Teichmüller space $\mathbf{T}$ and the base point of $\mathbf{T}$ equals $\tanh ^{-1} \hat{\varkappa}\left(\sigma_{a} \circ f\right)$.

(e) The curve $L=F\left(S^{1}\right)$ is a $k^{\prime}$-quasicircle with reflection coefficient $q_{L}=k_{0}$ connected with $k^{\prime}$ by

$$
\frac{1+q_{L}}{1-q_{L}}=\left(\frac{1+k^{\prime}}{1-k^{\prime}}\right)^{2}
$$

The quantity $\hat{\varkappa}(f)$ can be regarded as the outer limit Grunsky norm of $f$ on $\Delta$. 


\section{Remarks}

Passing to inversions $F_{f}(\zeta)=1 / f(1 / \zeta)$, this theorem can be reformulated for nonvanishing univalent function $F(\zeta)=\zeta+b_{0}+b_{1} \zeta^{-1}+\ldots, F(\zeta) \neq 0$ in the disk $\Delta^{*}$ with quasiconformal extensions to $\Delta$. For example, it holds for all odd univalent functions $F(\zeta)=\zeta+b_{1} \zeta^{-1}+\ldots$ in $\Delta^{*}$.

\section{Proof}

First of all, any quasiconformal extension of $f$ across the boundary circle $\partial \Delta$ into a domain containing $\bar{\Delta}$ can be extended, using Lehto's theorem [16], onto the whole sphere $\widehat{\mathbb{C}}$; then $\varkappa(f) \leq k(f)<1$, which yields $(a) \Rightarrow(b)$.

Conversely, any univalent function $f$ with $\varkappa(f) \leq k<1$ admits by the Pommerenke Zhuravlev theorem a $k_{1}(k)$-quasiconformal extension with $k_{1} \geq k$, hence $(a) \Rightarrow(b)$. by $(c)$.

The main part of the proof is to establish that any $f \in S(\infty)$ has the distortion given

Observe that every Grunsky coefficient $\alpha_{m n}(f)$ of $f$ is represented as a polynomial of a finite number of its initial Taylor coefficients $a_{2}, \ldots, a_{s}$, hence it depends holomorphically on Beltrami coefficients $\mu$ of quasiconformal extensions of $f$ running over the ball $\operatorname{Belt}\left(\Delta^{*}\right)_{1}$. The same is true for the Grunsky coefficients of each $\mathcal{R}_{p} f$, which also are polynomials of $a_{2}, \ldots, a_{l}$. This implies the holomorphy of maps

$$
h_{\mathbf{x}, p}(\mu)=\sum_{m, n=1}^{\infty} \sqrt{m n} \alpha_{m n}\left(\mathcal{R}_{p} f^{\mu}\right) x_{m} x_{n}: \operatorname{Belt}\left(D_{r}\right)_{1} \rightarrow \Delta
$$

for any fixed $p$ and any $\mathbf{x}=\left(x_{n}\right) \in S\left(l^{2}\right)$. Note also that $\sup _{\mathbf{x} \in S\left(l^{2}\right)} h_{\mathbf{x}, p}(\mu)=\varkappa\left(\mathcal{R}_{p} f^{\mu}\right)$, and each norm $\varkappa\left(\mathcal{R}_{p} f^{\mu}\right)$ is a continuous plurisubharmonic function on the ball $\operatorname{Belt}\left(\Delta^{*}\right)_{1}$.

Moreover, in view of holomorphy of the map $\iota_{r}: \mu \mapsto \mu_{r}$ stated above, the corresponding functions $h_{\mathbf{x}, p}\left(\mu_{r}\right)$ and $\varkappa\left(\mathcal{R}_{p} \sigma_{a} \circ f^{(} \mu_{r}\right)$ have similar properties. (2).

One can deal with $f \in S(\infty)$, otherwise one needs to compose $f$ with the transform

First assume that the function $f$ admits quasiconformal extension to $\{1<|z|<\infty\}$ of Teichmüller type, i.e., with Beltrami coefficient

$$
\mu_{0}(z)=\left\|\mu_{0}\right\|_{\infty}\left|\psi_{0}(z)\right| / \psi_{0}(z)
$$

where the quadratic differential $\psi_{0}$ can have at most a simple pole at the infinite point,

$$
\psi_{0}(z)=c_{3} z^{-3}+c_{4} z^{-4}+\ldots
$$

If $c_{3} \neq 0, c_{4} \neq 0$, then, noting that $\hat{\varkappa}\left(\mathcal{R}_{2} f^{\mu_{0}}\right)=\hat{\varkappa}\left(f^{\mu_{0}}\right)$, one can start with the squared map $\mathcal{R}_{2} f^{\mu_{0}}$ whose defining quadratic differential is by (4) of the form

$$
\mathcal{R}_{2}^{*} \psi_{0}\left(z^{2}\right)=4\left(c_{3} z^{-4}+c_{4} z^{-6}+\ldots\right)
$$

and has at $z=\infty$ zero of even order. Not to complicate the notations, we assume that this holds for $\psi_{0}$ (hence in (11) $c_{3}=0$ ), and we only need to consider the case when $\psi_{0}$ has at least two zeros of odd order. 


\section{REFERENCES}

1. Ahlfors L. Remarks on the Neumann - Poincaré integral equation. Pacific J. Math., 1952, vol. 2, pp. 271-280. $162 \mathrm{p}$

2. Ahlfors L.V. Lectures on Quasiconformal Mappings. Princeton, Van Nostrand, 1966.

3. Earle C.J., Kra I., Krushkal S.L. Holomorphic motions and Teichmüller spaces. Trans. Amer. Math. Soc., 1994, vol. 944, pp. 927-948.

4. Gardiner F.P., Lakic N. Quasiconformal Teichmüller Theory. Providence, Amer. Math. Soc., 2000. 372 p.

5. Grunsky H. Koeffizientenbedingungen für schlicht abbildende meromorphe Funktionen. Math. Z., 1939, vol. 45, pp. 29-61.

6. Krushkal S.L. Grunsky coefficient inequalities, Carathéodory metric and extremal quasiconformal mappings. Comment. Math. Helv., 1989, vol. 64, pp. 650-660.

7. Krushkal S.L. Quasiconformal extensions and reflections, Ch. 11. Handbook of Complex Analysis: Geometric Function Theory, Amsterdam, Elsevier Science, 2005, vol. II, pp. 507553.

8. Krushkal S.L. Strengthened Moser's conjecture, geometry of Grunsky inequalities and Fredholm eigenvalues. Central European J. Math., 2007, vol. 5, no. 3, pp. 551-580.

9. Krushkal S.L. Generalized Grunsky coefficient inequalities and quasiconformal deformations. Uzbek Math. J., 2014, no. 1, to appear.

10. Krushkal S.L. Ahlfors' question and beyond. Annals Univ. Bucharest, 2014, vol. 4 (LXIII), no. 1, to appear.

11. Kruschkal S.L., Kühnau R. Quasikonforme Abbildungen - neue Methoden und Anwendungen. Leipzig, Teubner, 1983. 172 p.

12. Kühnau R. Verzerrungssätze und Koeffizientenbedingungen vom Grunskyschen Typ für quasikonforme Abbildungen. Math. Nachr., 1971, vol. 48, pp. 77-105.

13. Kühnau R. Quasikonforme Fortsetzbarkeit, Fredholmsche Eigenwerte und Grunskysche Koeffizientenbedingungen. Ann. Acad. Sci. Fenn. Ser. A.I. Math., 1982, vol. 7, pp. 383391.

14. Kühnau R. Wann sind die Grunskyschen Koeffizientenbedingungen hinreichend für $Q$ quasikonforme Fortsetzbarkeit? Comment. Math. Helv., 1986, vol. 61, pp. 290-307.

15. Kühnau R. Möglichst konforme Spiegelung an einer Jordankurve. Jber. Deutsch. Math. Verein., 1988, vol. 90, pp. 90-109.

16. Lehto O. An extension theorem for quasiconformal mappings. Proc. London Math. Soc., 1965, issue 1, vol. s3-14A, pp. 187-190. $374 \mathrm{p}$

17. Pommerenke C. Univalent Functions. Göttingen, Vandenhoeck \& Ruprecht, 1975.

18. Schiffer M. Fredholm eigenvalues and Grunsky matrices. Ann. Polon. Math., 1981, vol. 39, pp. 149-164. 